\title{
Evaluation of Associated Gas Injection in the Miller Field
}

Moulds T. P.

BP Exploration, U. K.

Copyright 1995, Steering Committee of the European IOR - Symposium.

This paper was presented at the 8th. European IOR - Symposium in Vienna, Austria, May 15 - 17, 1995

This paper was selected for presentation by the Steering Committee, following review of information contained in an abstract

submitted by the author(8). The paper, as presented has not been reviewed by the Steering Committee.

\begin{abstract}
The Miller Field, located in the UKCS, contains a light oil with $18 \% \mathrm{CO} 2$ and a high GOR of $1830 \mathrm{scf} / \mathrm{stb}$. The associated gas is currently exported with $22 \% \mathrm{CO} 2$ content. The associated gas is multicontact miscible with the reservoir oil and so the potential exists to reinject the gas as a solvent for EOR. The potential for associated gas injection (AGI) was recognised prior to development of the field and the platform was constructed with sufficient space to accommodate AGI facilities and so minimise retro-fitting costs. The decision to implement AGI was deferred in order to gather further data after the field was on stream (first oil was June 1992)

The potential rewards from reinjecting the associated gas are evaluated in this paper. An integrated study is carried out involving the development of a compositional fluid description and reservoir characterisation through use of stochastic methods. At each stage of the evaluation appropriate simulation models are developed and where possible simplifications are introduced. The simulation work includes 1D modelling of phase behaviour and fine grid 2D and sector models to identify key reservoir uncertainties and optimise wateraltemating-gas (WAG) operating strategy at individual wells. AGI is then
\end{abstract}

introduced in the coarse grid Full Field model to ensure consistency with the ongoing waterflood.

The potential incremental recovery from a WAG scheme is quantified and the risks to delivery of the incremental oil are identified. The reservoir study is fully integrated with well engineering and facilities design. This approach shows the balance of risk and reward for AGI and provides the basis for the decision on whether to proceed with the scheme.

\section{Introduction}

The Miller field was discovered in 1982 , it lies in blocks $16 / 7 \mathrm{~b}$ and $16 / 8 \mathrm{~b}$ (Figure 1). The Miller reservoir is composed of Upper Jurassic submarine fan sandstones of the Brae Formation. It lies at a depth of $3970 \mathrm{~m}-4090 \mathrm{mss}$, covering an area of $45 \mathrm{sq} . \mathrm{km}$. with a maximum vertical relief of $120 \mathrm{~m}$. It is a combined structural and stratigraphic trap, the sandstones pinching out updip to the northwest. The initial OWC was at $\mathbf{4 0 9 0}$ mss. Reservoir porosity is approximately $14 \%$ and permeability to oil averages around $170 \mathrm{mD}$ but with higher permeabilities of up to $2000 \mathrm{mD}$ in the uppermost reservoir sands. The development was based on a STOIIP of 639 mmstbo and reserves of 301 mmstbo. This has subsequently been revised, at end 1994 STOIIP was 571 


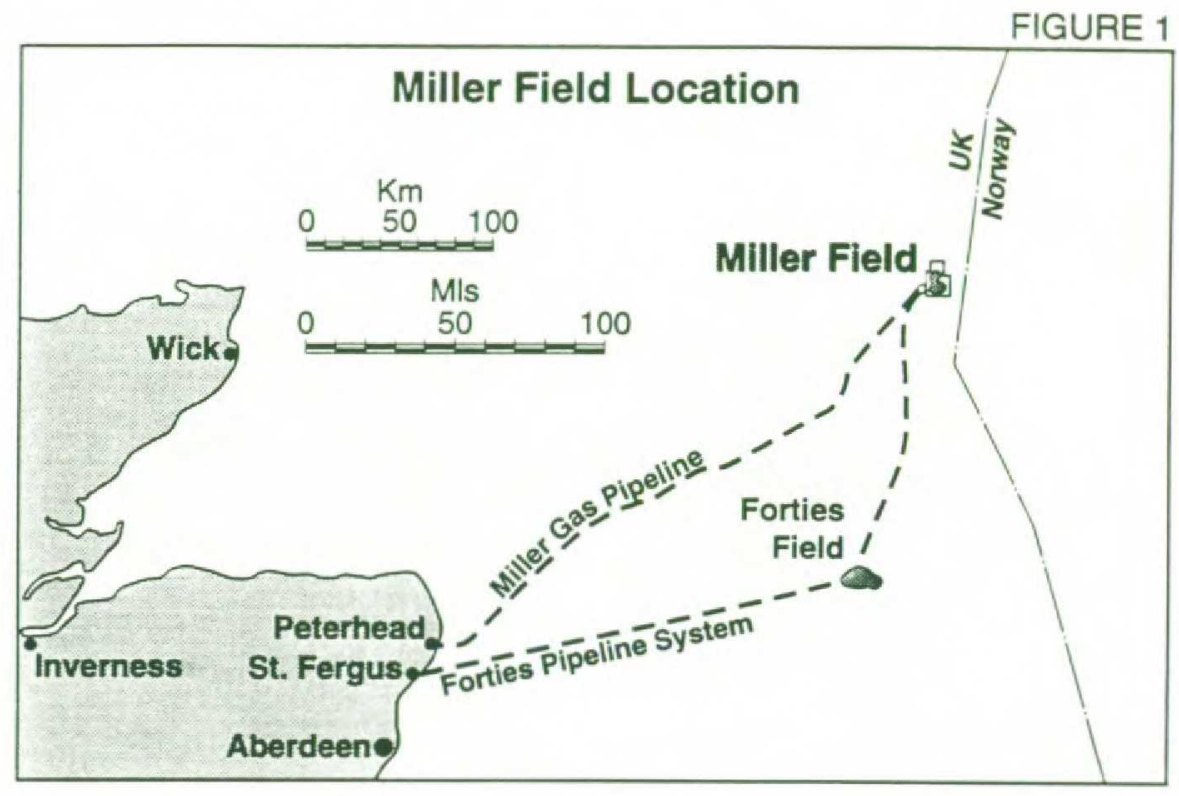

mmstbo and reserves 265 mmstbo.

The reservoir is an undersaturated oil reservoir with a saturation pressure of 4650 psia on average. Initial reservoir pressure was 7315 psia at the OWC, measured in well $16 / 8 \mathrm{~b}-3$. Subsequent to South Brae start-up in July 1983, appraisal data indicated Miller pressure depletion. At Miller start-up the average reservoir pressure was 6633 psia: this has declined further to around 5900 psia since start-up. Water injection and limited aquifer support has essentially maintained this pressure

The Miller development strategy is based initially on a line drive waterflood in the south-east of the field, achieving voidage replacement at an early stage to maintain production potential and sweep. The flood is then developed fieldwide into a peripheral waterflood thereby optimising field production and reserves. Production commenced on 8th June 1992 and water injection in August 1992. Production performance has been excellent with around $40 \%$ of reserves recovered since start-up and, based on modelling, in excess of $70 \%$ of reserves are expected to be recovered by the end of the plateau period.

The field is expected to remain on plateau for about 5 years, until the first half of 1997 , at average rate of $127 \mathrm{mstb} / \mathrm{d}$, and continue producing beyond the year 2000 .

\section{EOR Potential}

The anticipated waterflood efficiency of $80 \%$ in Miller is high but expected recovery is only $48 \%$ of STOIIP due primarily to the high residual oil saturation (35\%). Because of this the Miller reservoir was identified at a pre-development stage as having potential for an EOR scheme

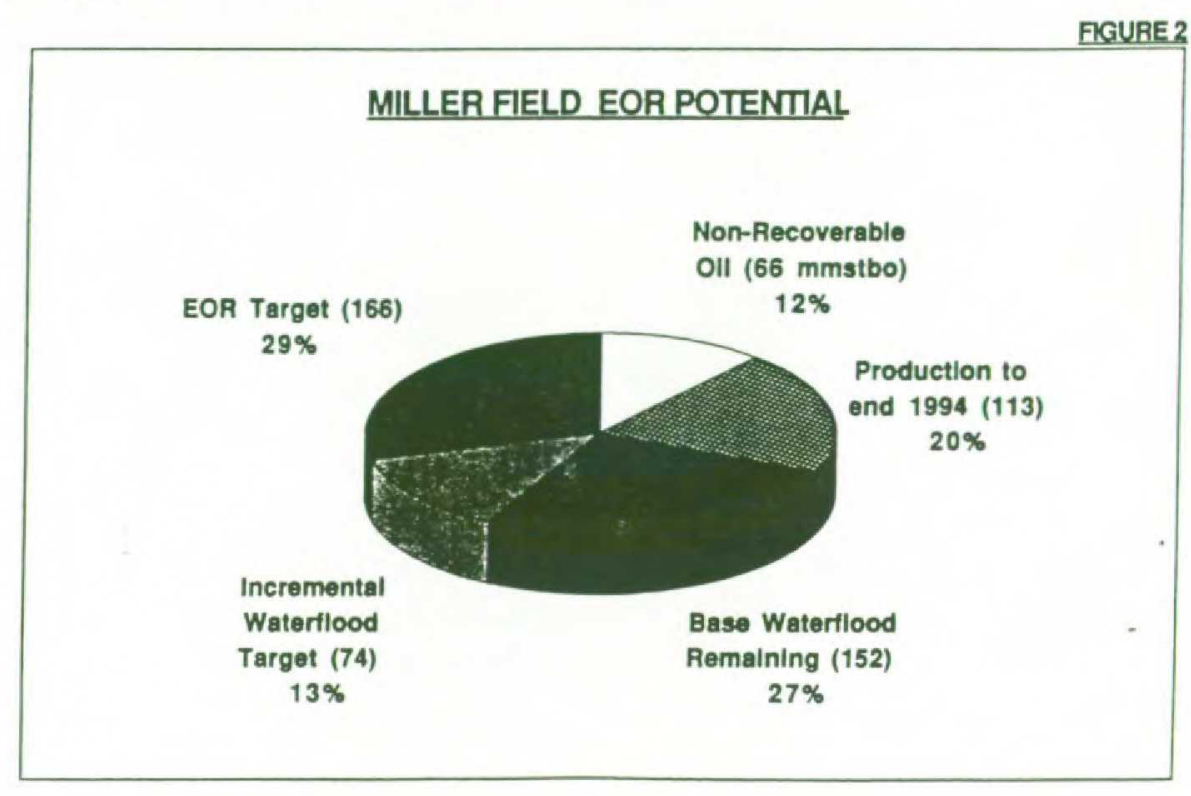


FIGURE 3

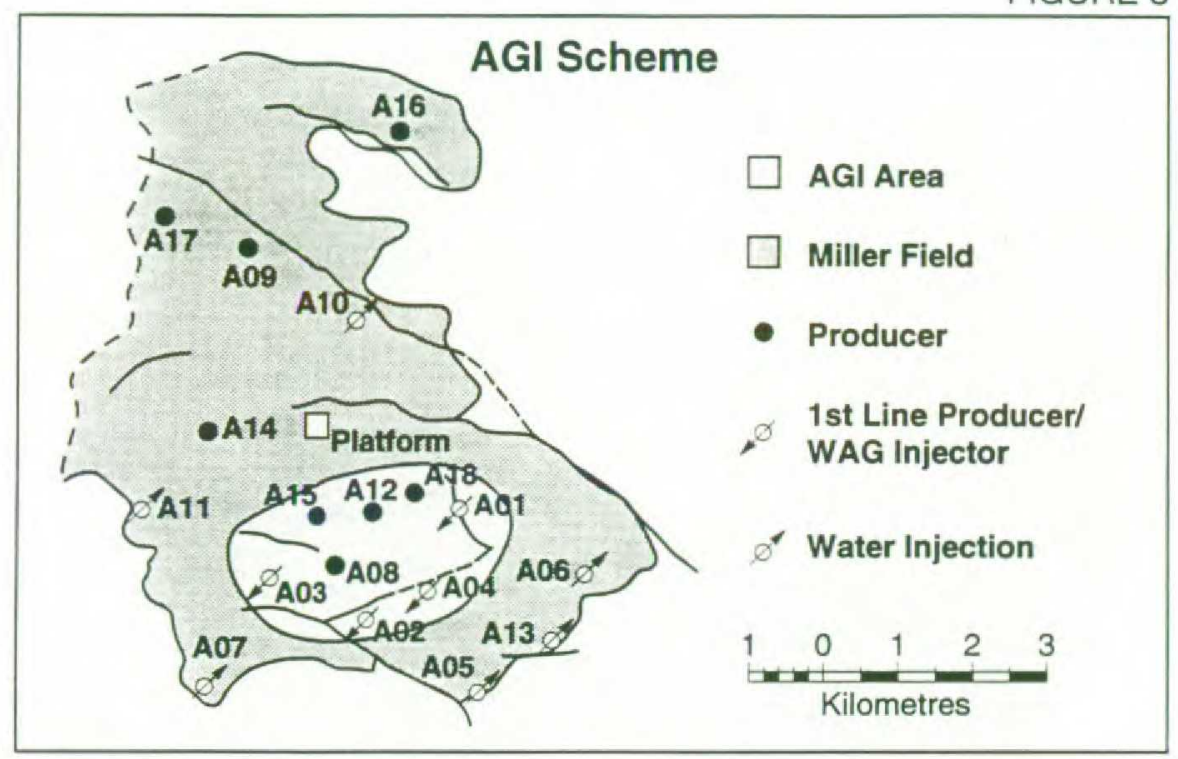

based on miscible gas flooding.

Based on 571 mmstbo STOIIP, Figure 2 shows the current reserves resource for Miller from secondary and tertiary recovery processes that may be applied during the life of the field (Swi 14\%, Sorw $35 \%$ and Sorm 10\%). The diagram shows that the maximum theoretical reserves from the Base waterflood is 339 mmstb. The maximum theoretical reserves based on tertiary gas injection is 166 mmstb (ie the EOR target). The immobile oil that cannot be recovered by either process is 66 mmstb.

The anticipated waterflood efficiency in the current plan is already high. Based on a comparison with other UKCS fields it is unlikely that recovery by waterflood can be improved to greater than 52\% STOIIP. The large tertiary oil target (the EOR target) is clearly an important resource to increase Miller reserves. Understanding the potential exploitation of this resource is extremely important to the field life of Miller.

\section{AGI Scheme}

There is a limited window of opportunity to implement an AGI scheme on Miller. There are constraints on the earliest and the latest times at which the scheme can be initiated.

The earliest time at which AGI can be implemented is determined by progress of the waterflood. Screening studies showed that the most productive region in which to apply AGI is the core south-east area of the field. WAG injection is carried out by turning around the first line production wells after they have watered out (Figure 3). Injection cannot start until all waterflood recoverable oil has been swept past these wells and, based on simulation, an adequate buffer of water is established ahead of the WAG injectors to safeguard the base waterflood reserves.

The latest time at which the scheme can be started is determined by the availability of sufficient associated gas to provide the necessary gas injection volumes. The efficient waterflood leads to a rapid decline in oil rate when the field comes off plateau. The AGI scheme will only produce sufficient oil to be economic if it can make use of the gas which is available from the end of the plateau onwards.

The combination of these two constraints leads to an optimum start-up time for AGI of $1 \mathrm{Q} 97$, just prior to the end of plateau.

\section{Compositional Model}

Miller requires compositional support because it is volatile and has high $\mathrm{CO}_{2}$ content $(\approx 18 \mathrm{~mol} \%)$. A reference equation of state (EOS) was developed which uses 15 components, individual components were used through $\mathrm{C}_{6}$ and Whitson's technique ${ }^{1}$ was used to generate $\mathrm{C}_{7+}$ pseudocomponents. The reference EoS was matched to static PVT data within experimental uncertainty, it was also matched to experimental slimtube measurements. 


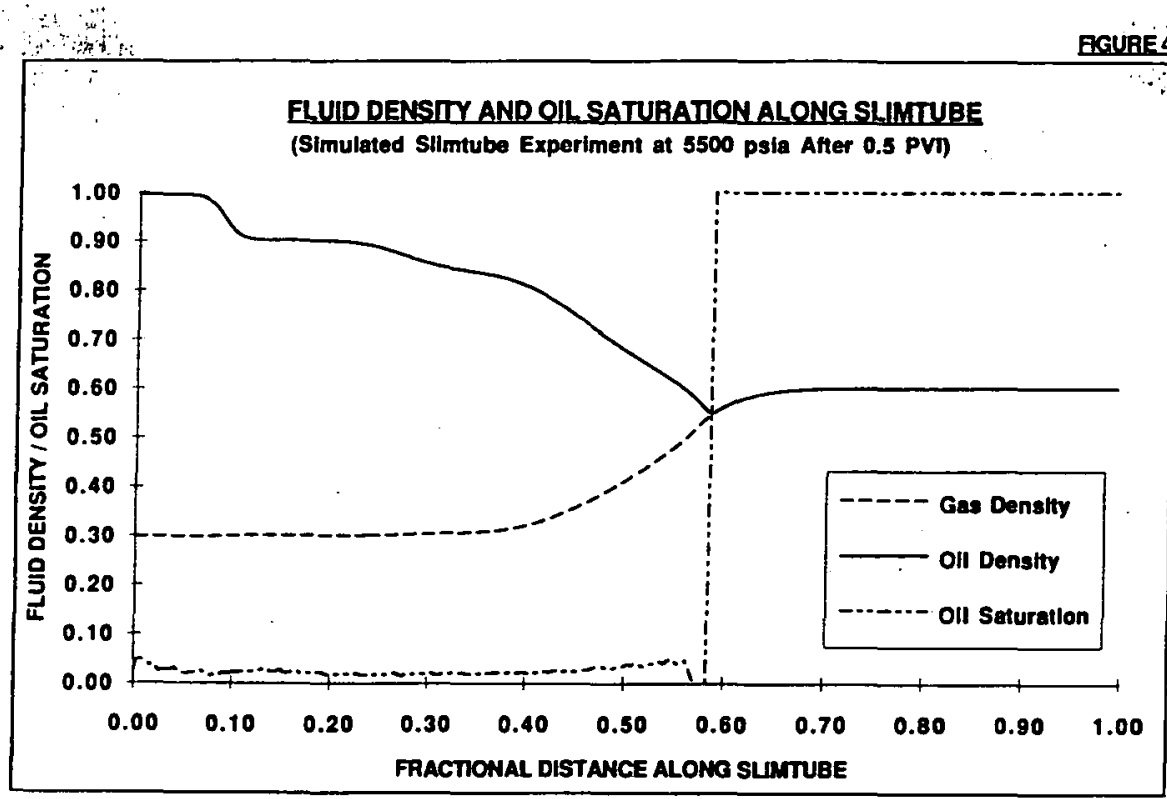

Slimtube simulation modelling using a 1D model showed the minimum miscibility pressure (MMP) to be 5050 psia. This is at the lower limit of predicted operating conditions and so it is expected that the associated gas will achieve miscibility.

The 1D simulation work provides information on the phase behaviour. Miscibility is found to be achieved by multiple contact between the injected gas and reservoir oil. As the gas front approaches the light components condense from the gas phase into the oil and the mole fraction of the light components in the liquid phase increases. This is followed by vaporisation of intermediate components from the oil into the gas phase. Figure 4 shows the gas density increasing sharply near the front, the gas and oil densities become identical, giving rise to a thermodynamically miscible displacement.

The reference EoS provides a reliable means of predicting the phase behaviour of Miller but, with 15 components, it is unwieldy to use in reservoir simulation. An 8 component "lumped" EoS was developed for simulation purposes. The components in this EoS each represent several components in the reference EoS. The lumping scheme for the simulation EoS was chosen by means of the Newley and Merrill method ${ }^{2}$. Repeating the slimtube simulation work confirmed that the reference and reduced component EoS formulations produced the same results.

A necessary check when running compositional models is to ensure that numerical dispersion effects remain small. Throughout the 1D work the quality of the results was checked by determining the degree of numerical dispersion as a function of grid size.

\section{D Models}

2D models allow the effects of gravity on process performance to be evaluated. For this reason they are especially valuable for the analysis of gas displacement". processes. The issues which were addressed using the 2D cross section models included perforation strategy, compositional effects and the impact of reservoir description on vertical sweep.

To evaluate the feasibility of miscible gas injection requires that the WAG process is simulated taking full account of compositional effects and with a realistic reservoir description. The reservoir description for the Miller AGI models was developed using geostatistical methods.

\section{Reservoir Description}

For reservoir management purposes the Miller reservoir is divided into five oil bearing zones. Across much of the reservoir the zones are separated by substantial shales. The coverage and continuity of these inter-zone shales varies, in some cases the shales are extensive and create significant pressure breaks between zones. In addition to the major shales there are also smaller shales within each zone. The net:gross ratio in the core area of the field is about $90 \%$ and so the intra-zone shales account for only a small fraction of 
the pore volume.

Reservoir descriptions in the crosssection models were based on stochastically generated permeability distributions conditioned to well data. In addition both the inter-zone and intra-zone shales were represented explicitly by transmissibility barriers.

\section{Permeability}

Core data was the preferred data source for the permeability description. Miller has good core coverage and the routine core data provide a valuable database of permeability variation. Preliminary simulation work indicated that a simulation grid of 56 layers would provide adequate definition of the WAG process and capture the required level of heterogeneity in the permeability description. As often occurs, it is a matter of compromise to use grid blocks which are sufficiently large that the total number of cells in the model do not lead to impractically long simulation times and at the same time the model must capture the variation in reservoir properties (heterogeneity). The typical layer thickness in the 56 layer model was $2 \mathrm{~m}$.

Horizontal permeability control data was calculated as a simple arithmetic average of core data at the wells. This is appropriate as the horizontal permeability correlation length is substantially greater than the length of the cells.

Vertical permeability is more difficult to define. Geostatistical analysis of the routine core data showed that there was no correlation between consective vertical permeability data points (typically separated by $1 \mathrm{~m}$ ). Thus it is not appropriate to characterise the fine grid model vertical permeability for a $2 \mathrm{~m}$ thick model layer on the basis of one or two uncorrelated core data points. Finer scale evaluation is required to take account of the rapidly changing permeability in the vertical direction

Miller is fortunate in having minipermeameter data available. The minipermeameter obtains permeability readings at $5 \mathrm{~cm}$ intervals. Geostatistical analysis of this data confirmed that there is no correlation between permeability data points which are more than 1m apart. For readings less than $1 \mathrm{~m}$ apart there is an increasing level of correlation as the distance between readings decreases.

The $\mathrm{kv} / \mathrm{kh}$ ratio for the fine grid model was obtained by combining the results of both routine core analysis, which provide a direct measure of $\mathrm{kv}$ and $\mathrm{kh}$ at the same depth, and minipermeameter data which provide information on how rapidly kh is changing for points which are only $5 \mathrm{~cm}$ apart. The outcome of the analysis was that the $\mathbf{k v} / \mathrm{kh}$ ratio for each fine grid model cell should be assigned as a random selection from a uniform probability distribution between 0.15 and 0.4 . For comparison, the average $\mathrm{kv} / \mathrm{kh}$ ratio of a core plug is 0.8 .

Explicit transmissibility barriers were input to represent the shales. The major inter-zone shales were conditioned to the wells, the smaller intra-zone shales were entered as a random distribution of transmissibility barriers.

Using a numerical pressure solution it was found that for P10/P50 / P90 reservoir descriptions the effective kv/kh ratio within each reservoir zone $0.05 / 0.1 / 0.2$, this includes the effect of the intra-zone shales.

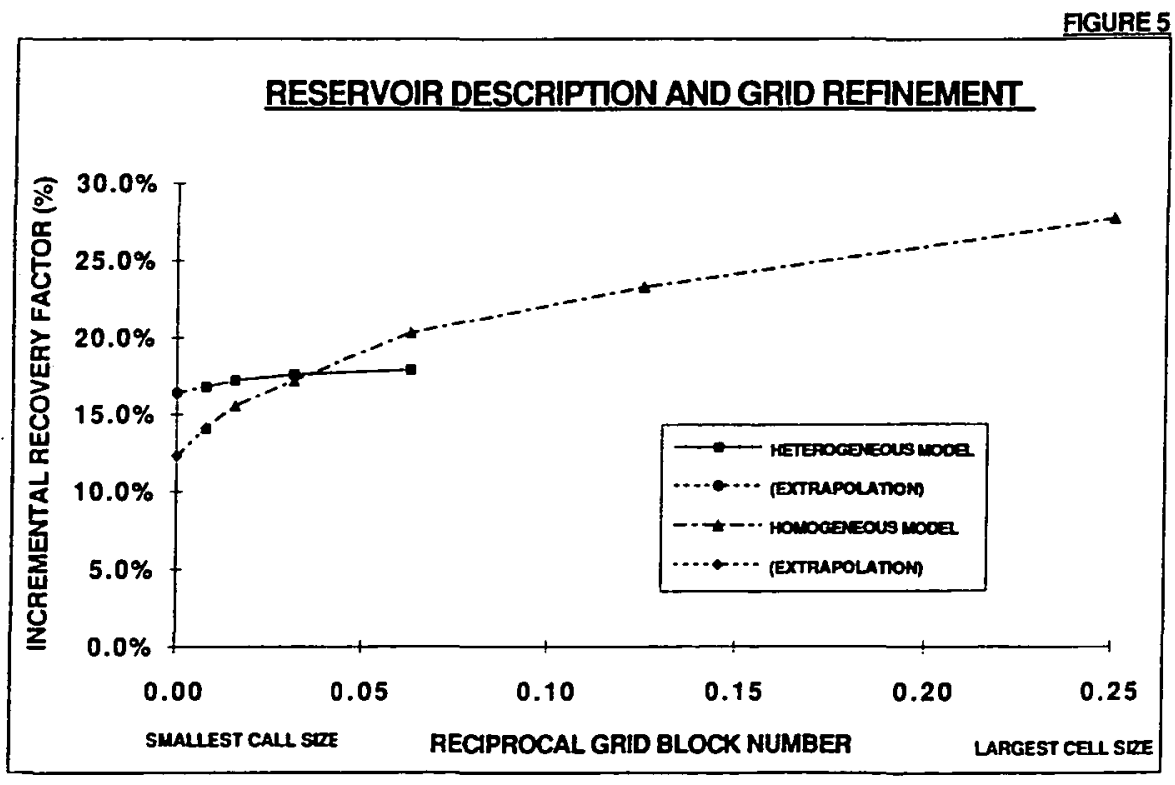




\section{Grid Sizé Sensistivity}

One of the primary concerns in miscible gas simulation is that inappropriate grid size may result in significant errors in the incremental recovery predicted for WAG compared to the base waterflood recovery.

Grid refinement effects are often carried out using homogeneous models. It was found that the more realistic reservoir description used in this study was far less sensitive to grid refinement that a homogeneous model with the same average properties. Figure 5 shows the sensitivity of incremental recovery to grid refinement in both heterogeneous and homogeneous reservoir descriptions. The 2D cross section grids in this study correspond to a reciprocal grid block number of 0.04 . The heterogeneous model simulation results are sufficiently accurate to be used without further modification for the effects of grid refinement.

\section{Perforation Strategy}

A major concem with gas injection in the Miller reservoir is the potential for gas override. The upper reservoir zone (zone J) contains high permeability sands, these have been observed at several wells but are not considered to be correlatable between wells. The concern is that gas will segregate rapidly at the injection well, migrate into these high permeability sands and override the target waterflood residual oil.

For a cross section containing a moderate amount of high permeability sand then zone $J$ perforation of either the injection or production well resulted in a loss of about $20 \%$ of the incremental reserves. Zone $J$ perforation of both the injection and production well results in a loss of $40 \%$ of the incremental reserves. Isolation of zone $J$ at the injection and production wells is a key well completion issue which is critical to the success of AGI.

Unfortunately simpiy not perforating zone $\mathrm{J}$ does not guarantee that gas is not injected directly into it at the injection well. During the WAG water injection phase it is expected that thermal fractures will be created at the injection wells. The fractures will heal to some extent during gas injection but, having once fractured into a zone, it is unwise to assume that the fracture will heal completely. Numerical modelling using a thermal simulator was used to estimate the likely location of thermal fractures during gas and water injection phases. The perforation strategy at each well was adapted as necessary to minimise the risk of thermal fracturing into zone J.

\section{Compositional Impact in 2D}

The 2D cross section modelling was carried out using the 8 component EoS. Component transfer between the phases results in the gas becoming much heavier at the front such that the oil and gas density and viscosity are almost identical. An important consequence of the component transfer is that there is minimal gravity difference between the gas and oil at the front. This stabilises the flood and leads to much more efficient vertical sweep than would otherwise occur. This is particularly important where a degree of gas override has occurred and the injected gas has broken through the water buffer and contacted unswept oil. In this situation the minimal gravity difference between the miscible gas and oil results in a relatively efficient vertical sweep. To a great extent this mechanism provides a safeguard against early gas breakthrough.

\section{Waterflood Performance}

The timing of WAG start-up must take into account the position of the waterflood front. If the front was too near the injection well then the injected gas rapidly contacts unswept oil. Although the gas and oil mix to create a miscible displacement the gas remains more mobile. This leads to a situation at gas breakthrough where a significant volume of oil remains unswept by either gas or water. The simulator shows that ultimately, with an extra year of WAG injection, similar incremental recovery is achieved. In this situation the WAG displacement is less efficient and reservoir management more difficult. The optimum position for the waterflood at the start of WAG injection appeared to be half way between the injector and producer.

\section{Reservoir Parameters}

It is important to understand the impact of different reservoir parameters on incremental recovery. In this way it is possible to identify where the greatest risks lie and whether they are associated 
with factors which can be managed. For instance, isolation of zone $J$ was an important issue but one which it is possible to manage, by comparison if recovery is sensitive to shale distribution there is very little that can be done to alter the outcome other than to be prepared to take remedial action.

The factors assessed using the 2D models included permeability and shale distribution, kv/kh ratio, fault density, relative permeability and residual oil saturation to miscible gas (Sorm). Further reservoir description sensitivity work was also carried out with fine grid 3D sector models. The simulation results for two of these parameters in particular are worthy of note: zone J permeability and its impact on gas override and $\mathrm{kv} / \mathrm{kh}$ ratio.

The potential for gas override depends on a combination of perforation policy, thermal fracturing, fault pattern and zone J permeability. It was found that if gas override occurs it can halve the incremental recovery. This is a major downside risk which highlights the need for careful well engineering.

The $\mathrm{kv} / \mathrm{kh}$ ratio is, classically, one of the key factors in the success of a WAG scheme. A low kv/kh ratio increases the ratio of viscous:gravity forces which leads to slower segregation of gas and water and a more effective vertical sweep. It was surprising to find that in Miller the impact of varying the $\mathrm{kv} / \mathrm{kh}$ ratio was at best insensitive and on occasions the incremental recovery was in the reverse order to that expected. Examination of the displacement process shows that:

due to compositional effects there is very little difference between the oil bank and miscible gas density at the interface.

low $\mathrm{kv} / \mathrm{kh}$ extends the WAG cone further from the injection well as predicted in classical analysis.

higher $\mathrm{kv} / \mathrm{kh}$ leads to less of the mobilised oil being trapped below shales.

different $\mathrm{kv} / \mathrm{kh}$ assumptions can alter the sweep pattem between reservoir zones

The relative impact of each of these factors determines the effectiveness of the WAG flood. In Miller the impact of $\mathrm{kv} / \mathrm{kh}$ values between 0.05 and 0.2 produces a difference of less than $10 \%$ in the incremental reserves.

\section{D Fine Grid Models}

In order to use the simulation results for economic evaluation it is necessary to include the impact of 3D geometry. The 2D grid refinement work had shown that there was scope to increase the grid size without incurring significant deterioration from numerical dispersion. In addition, the 2D simulation work had found that much of the section (behind the injection well and in the deeper zones) had minimal impact on WAG performance. These factors showed that there was scope to create fine grid 3D (sector) models which would model the area between a WAG injection and production well without compromising on grid size.

Two sector models were constructed using analogous reservoir description methods to the 2D models.

The sector models provide a direct comparison of 2D and 3D sweep efficiency. In addition they were also used to optimise the WAG operations and to evaluate further reservoir parameters.

The sector model indicated an areal sweep of about $70 \%$ in the region between the injector and producer. The sweep varied greatly between zones and in all cases there was evidence of gas underrunning the major inter-zone shales. The effects of component transfer between the phases reduced the gas mobility at the front and, like vertical sweep, improved areal sweep. In particular there was little evidence of gas channelling between the injection and production well.

\section{WAG Optimisation}

The sector models are the most appropriate means to estimate WAG efficiency and determine the optimum WAG ratio and duration. WAG performance was assessed in terms of both incremental oil recovery and net gas efficiency (net reduction in sales gas / incremental oil volume). These two performance measures are combined in Figure 6. Ideally a complete set of simulation runs would be carried out to fully describe all the curves on the figure, but even with the available data the diagram gives a clear indication of the most attractive schemes. The figure summarises the key performance measures and indicates which cases are likely to be the most economically attractive, ie highest 


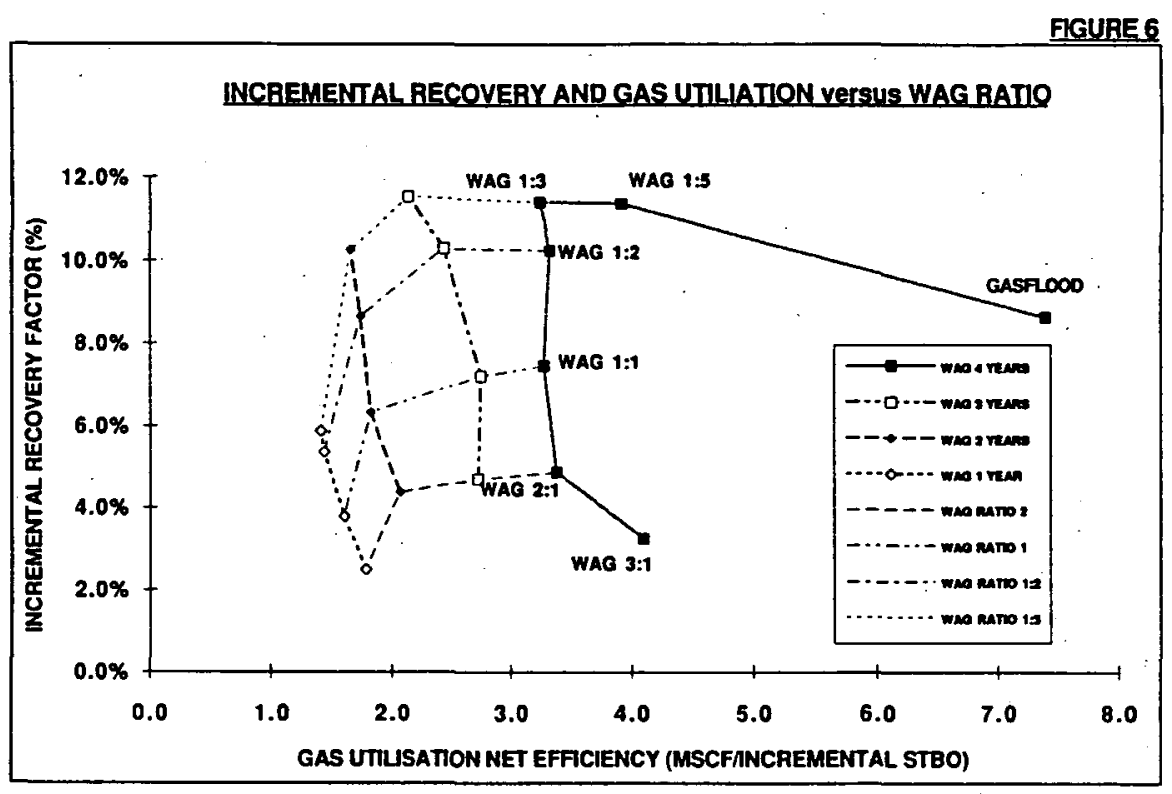

recovery and lowest $\mathrm{mscf} / \mathrm{bbl}$. Conclusions which can be drawn from the figure are:

One year of WAG injection fails to capture much of the potential incremental oil.

Recovery factor and gas utilisation improve with lower WAG ratio down to a ratio of $1: 3$.

In Miller the optimum strategy is to inject gas at the highest practical gas-towater ratio for a period of between 2 and 4 years in each pattern. In light of the greater efficiency at low WAG ratio and the limited number of potential WAG injection sites in Miller, the preferred strategy was to focus on a few areas with greatest potential. For Miller, a fieldwide scheme at high WAG ratio is inappropriate.

\section{Full Field Simulation Study}

To fully evaluate AGI in the context of future Miller development it is necessary to simulate the scheme on the full field simulation model to determine:

AGI impact on the planned waterflood development

Quantify the availability of gas for injection

In order to carry out these studies a link must be established between the full field and the fine grid sector model performance. This was done by upscaling the sector model to the same grid dimensions as the full field simulator. The fluid description was recast in terms of a Todd-Longstaff modified black oil model and the performance of the compositional fine grid and the coarse grid model were matched. The matched coarse grid sector model fluid properties were then taken into the full field model.

The role of the coarse grid full field model is necessarily limited in this type of study. The model is adequate to describe the overall AGI recovery provided the scheme and recovery processes do not vary greatly from those on the fine grid sector models. However, the full field model, which represents each zone by a single layer, is too coarse to carry out reservoir description sensitivites. Its purpose in the AGI evaluation was principally to ensure that an overall gas balance was achieved for the field and to estimate the impact of a shortfall in gas on AGI incremental recovery.

\section{Reserves Distribution}

The decision making process as to whether to proceed with AGI was based on a probabilistic outcome of incremental reserves. The input data for the uncertainty analysis was taken from the simulation results which quantified the impact of individual reservoir parameters on incremental reserves.

Uncertainty was quantified by using the parametric method 3 to combine the uncertainty from the various reservoir parameters into a single reserves probability function. The parametric method requires that the impact of each of 
FGURE 7

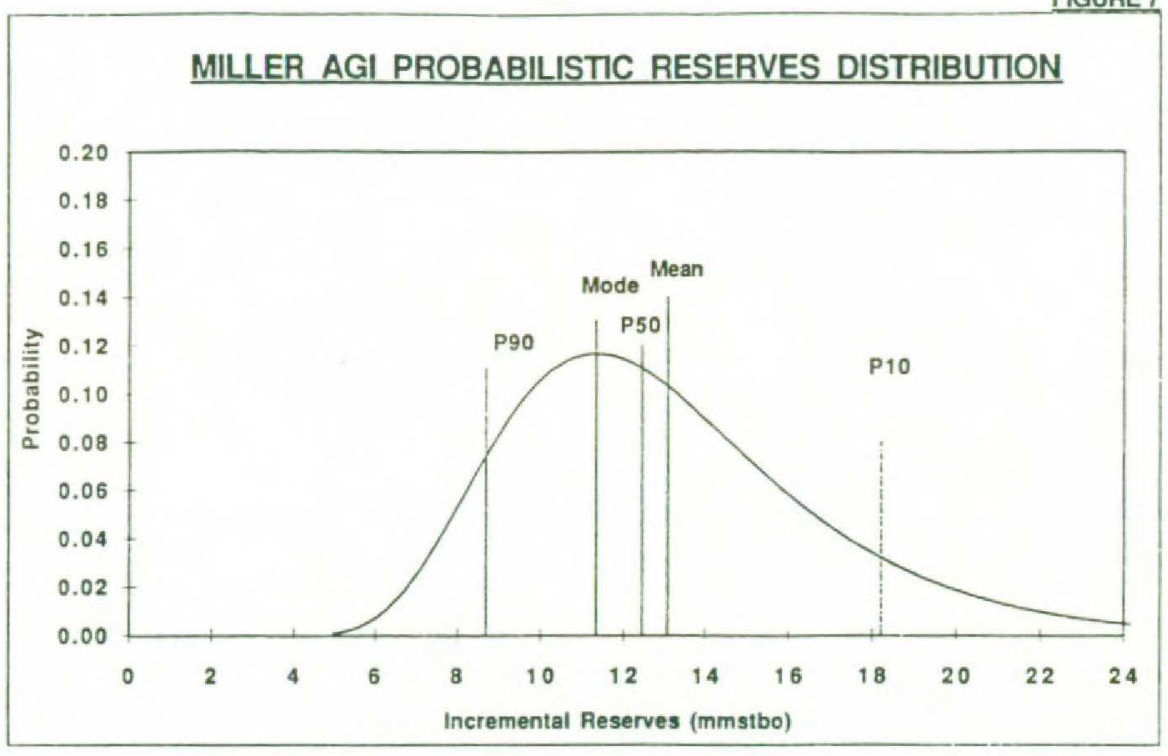

FIGURE 8

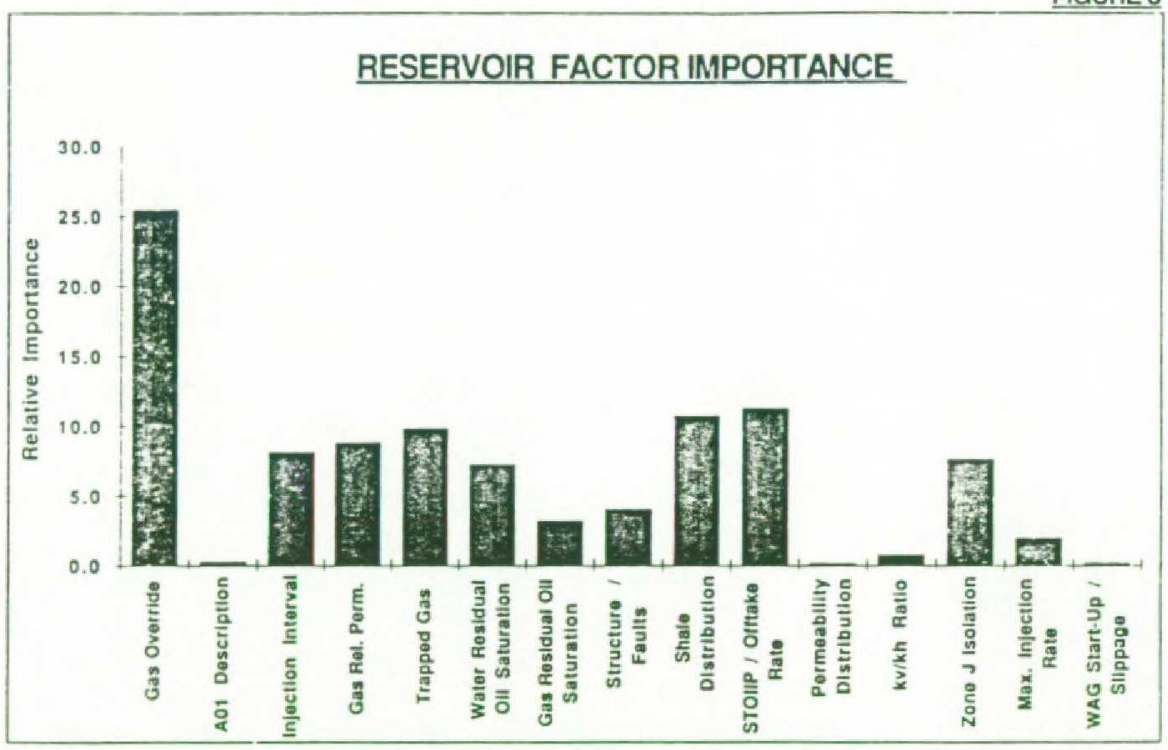

FIGURE 9

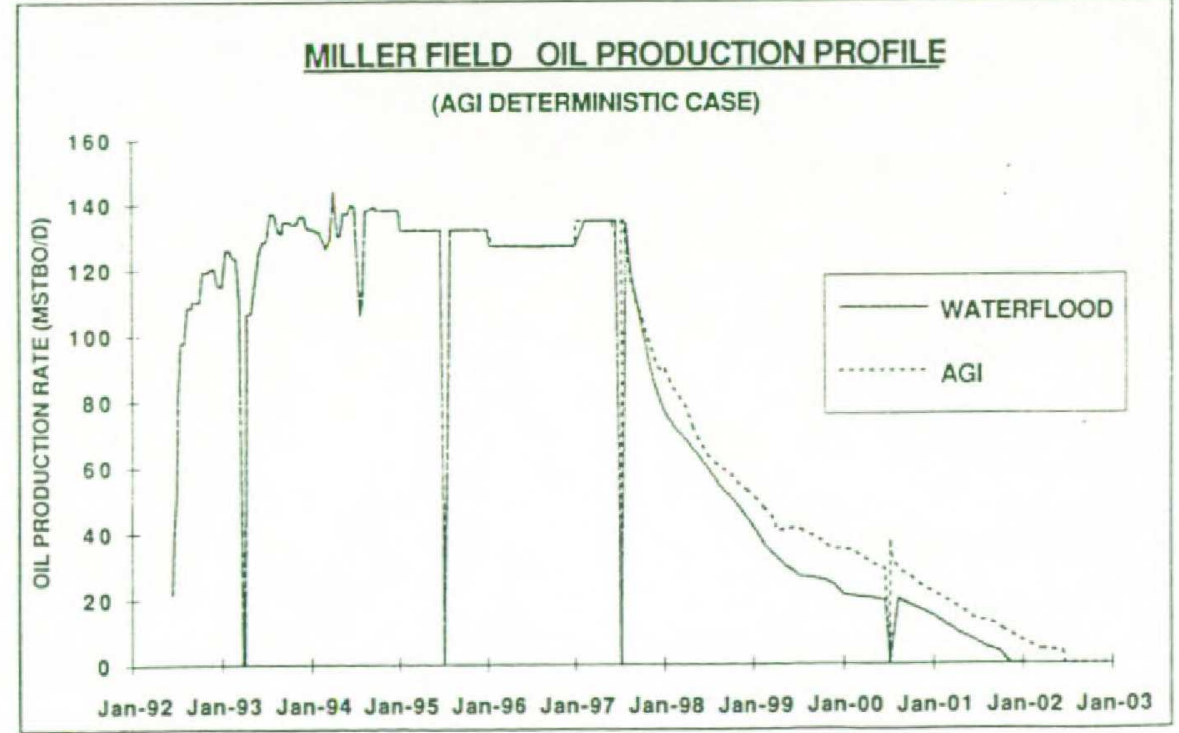


the key reservoir parameters on increméntal recovery can be described in terms of a probability distribution. In fact it is only necessary to define the mean and variance of the input distributions and these can be estimated from the deterministic models results. The resultant probabilistic AGI incremental reserves distribution is shown in Figure 7.

An additional outcome of the probabilistic approach is that the relative risk associated with each reservoir parameter is calculated as part of the analysis. Figure 8 shows the relative importance of each reservoir parameter to the overall uncertainty.

\section{Production Profiles}

In general it is useful to describe uncertainty in terms of the P10, P50 (or mean) and P90 reserves. In order to carry out an economic analysis these reserves must also be accompanied by production and injection profiles. The risk analysis provides guidance on the construction of suitable profiles. The P50 and mean cases are not dissimilar from the deterministic base case (Figure 9). It is relatively simple to prorate the deterministic results to generate the required reserves although some iteration is required to ensure that the overall gas balance is maintained.

The reservoir factor importance work identified the main factors which contribute to upside and downside reserves. The greatest downside is associated with gas override and the upside is most likely to occur due to higher STOIIP in the AGI target area This information provides the opportunity to ensure that the P10 and P90 profiles properly represent those factors which are responsible for the upside or downside. In particular, attention should be given to deterministic cases which are close to the upside and downside rather than simply prorating the most likely deterministic cases. The downside production profile should reflect gas override, ie early gas breakthrough and gas injection stopping earlier than planned after the problem is identified. Similarly the upside case should reflect higher STOIIP and longer breakthrough time. In these cases it is important to view WAG recovery against comparable waterflood cases.

The probabilistic reserves description together with appropriate production profiles provide the most complete technical information on which to assess the balance of risk and reward for AGI. The data provided by this analysis forms a basis for the decision on whether to proceed with an AGI scheme.

\section{Conclusions}

1) An integrated reservoir study of a miscible gas injection scheme has demonstrated a significant IOR target in the Miller field.

2) The benefits from miscible gas injection are presented as a reserves probability function together with appropriate production profiles.

3) Key risks associated with miscible gas flooding in Miller are identified. The risks are quantified through parametric uncertainty analysis applied to deterministic results from appropriately scaled simulation models.

\section{Acknowledgements}

The author wishes to thank the management of BP Exploration (operator of Miller Field) and the working interest owners: Enterprise, Conoco and Sante-Fe for permission to publish this work.

\section{References}

1) Whitson, C.H., Anerson, T.F. and Srøeide, I., "C7+ Fraction Characterisation", Chom and Mansoori eds., Taylor \& Francis, (New York, 1989), 35-56.

2) Newley, T.M.J., and Merrill, R.C., "Pseudocomponent Selection for Compositional Simulation", SPE Reservoir Engineering, November $1991,490-496$.

3) Smith, P.J., Hendry, D.J., and Crowther, A.R., "The Quantification and Management of Uncertainty in Reserves", SPE 26056, presented at Western Regional Meeting, Anchorage,USA, May 1993.

\section{SI metric conversion factor}

$\mathrm{bbl} * 1.589873 \mathrm{E}-01=\mathrm{m}^{3}$
$\mathrm{psi} * 6.894757 \mathrm{E}+00=\mathrm{kPa}$ 